\title{
INVESTIGATION OF PROTEOMIC BIOMARKERS IN IN VIVO HEPATOTOXICITY STUDY OF RAT LIVER: TOXICITY DIFFERENTIATION IN HEPATOTOXICANTS
}

\author{
Toshinori YAMAMOTO, Rie KIKKAWA, Hiroshi YAMADA and Ikuo HORII \\ Worldwide Safety Sciences, Pfizer Global Research \& Development, Nagoya Laboratories, Pfizer Inc, \\ 5-2 Taketoyo, Aichi 470-2393, Japan
}

(Received September 7, 2005; Accepted October 31, 2005)

\begin{abstract}
We investigated the overall protein expression profiles in the in vivo hepatotoxicity of rats induced by four well-recognized hepatotoxicants. Acetaminophen (APAP), amiodarone (AMD), tetracycline (TC) and carbon tetrachloride (CTC) were administered to male rats by gavages and the liver at $24 \mathrm{hr}$ post-dosing was applied to the proteomic experiment. Blood biochemistry and histopathology were examined to identify specific changes related to the compounds given. Protein expression in the liver was investigated by 2-dimensional gel electrophoresis (2DE), and spots showing a significantly different expression in treated versus control group were excised from gels and identified by Q-Tof mass spectrometer. They were well characterized based on their functions related to the mechanisms of toxicity of the compounds. Among them, we focused on the 8 proteins that were affected by all 4 compounds examined. Proteins related to oxidative stress response such as carbonic anhydrase III (CA3) and 60kDa heat shock protein (HSP60), and energy metabolism such as adenylate kinase 4 (AK4) were found. Moreover, hierarchical clustering analysis using 2D-gel spots information revealed the possibility to differentiate the groups based on their toxicity levels such as severity of liver damage. These results suggested that assessing the effects of hepatotoxicants on protein expression is worth trying to screen candidate compounds at the developmental stage of drugs.
\end{abstract}

KEY WORDS: Liver, Hepatotoxicants, Proteomics, Pattern recognition, Hierarchical clustering, Oxidative stress

\section{INTRODUCTION}

In the early stage of drug discovery, toxicity assessment at the molecular level, namely the molecular toxicological approach, has been demanded in context with high throughput safety screening. Several drugs were obliged to be withdrawn from the marketplace or labeled for severely limited usage for causing unpredictable idiosyncratic liver failure in nonclinical and clinical stages (Chitturi and George, 2002; Maddrey, 2005; Watkins, 2005). Depending on susceptibility, many drugs, such as bromfenac and troglitazone, were reported as unpredictable cases even though the effects of drugs on the liver were well assessed at the screening stage. Bromfenac (Duract), a nonsteroidal anti-inflammatory drug (NSAID), was introduced to the market in 1997 , and was withdrawn voluntarily from clinical use in 1998 for causing severe liver injury associated with long-term use (Hunter et al., 1999; Moses et al., 1999; Rabkin et al., 1999). Another example was the case of troglitazone. In 1997, troglitazone (Rezulin or Noscal) was approved and introduced to market as a new class of oral antidiabetic drug for type II diabetes mellitus. Several cases of severe hepatocellular damage, hepatic necrosis and hepatic failure, were reported shortly after (Gitlin et al., 1998; Vella et al., 1998; Herrine and Choudhary, 1999; Faich and Moseley, 2001), which also resulted in withdrawal from the marketplace in 2000. These examples revealed that the standard and conventional approaches in preclinical toxicity screening, which included toxicity studies in non-clinical and clinical studies, resulted

Correspondence: Toshinori YAMAMOTO (E-mail: Toshinori.Yamamoto@pfizer.com) 


\section{T. YAMAMOTO et al.}

in a failure to detect the potential for drugs to produce idiosyncratic liver injury. Therefore, new biomarkers for molecular toxicological approach are required to detect a relevant hepatotoxicity to human.

Recently, the development of techniques to investigate genomics/transcriptmics using DNA microarray, proteomics using two-dimensional gel electrophoresis (2DE) and mass spectrometry (MS), and metabonomics using nuclear magnetic resonance (NMR) have enabled us to investigate changes in biological events at the molecular level (Nicholson et al., 1999; Hayes and Bradfield, 2005). There is no doubt that investigation on mechanisms of toxicity and developments of brand-new biomarkers using these technologies will contribute to molecular toxicological approach and high throughput safety screening in the future (Bandara and Kennedy, 2002; Henry, 2003; MacGregor, 2003). Particularly proteomics, which allows the investigation of protein expression, can provide information on disease processes and mechanisms of drug action at the level of protein functions in the cells (Pennington and Dunn, 2001). Combination of clinical pathology data and histopathological findings with proteomics findings also offers new insights and understanding of the mechanisms of toxicity, and alterations relevant to toxicity that have not been previously reported might be found (Kennedy, 2002).

We have already reported the effects of acetaminophen (APAP), amiodarone (AMD) and tetracycline (TC) on oxidative stress-related proteins, such as glutathione peroxidase, peroxiredoxin 1 and 2, in primary rat hepatocyte cultures detected using the proteomics approach (Yamamoto et al., 2005). In the present study, we investigated the effects of carbon tetrachloride (CTC) and the above-mentioned 3 compounds on protein expression in vivo in the liver. The proteins showing a significantly different expression in treated groups were subjected to peptide sequence tags using Q-Tof mass spectrometry. APAP, AMD, TC and CTC have well-recognized mechanisms of hepatotoxicity; namely, hepatic necrosis for APAP (Nelson, 1990), phospholipidosis for AMD (Poucell et al., 1984; Goldman et al., 1985), steatosis by accumulation of triglycerides for TC (Deboyser et al., 1989), and steatosis caused by fatty degeneration for CTC (Recknagel et al., 1989). Therefore, the use of these positive controls is considered to be suitable for this investigation.

In this paper, we demonstrated 8 liver proteins that were affected by all compounds tested. Some of them were related to the process of oxidative stress and $\beta$-oxidation of fatty acids. The results of pattern recognition analysis using protein expression profiles were well correlated with those of conventional toxicological findings.

\section{MATERIALS AND METHODS}

\section{Test compounds}

Acetaminophen (APAP) was purchased from Sigma (St. Louis, MO, USA). Amiodarone hydrochloride (AMD) was obtained from ICN Biomedicals Inc. (Aurora, OH, USA). Tetracycline hydrochloride (TC) and carbon tetrachloride (CTC) were purchased from Wako Pure Chemicals (Osaka, Japan).

\section{Chemicals and reagents}

Electrophoresis grade reagents [acrylamide, agarose, ammonium persulphate (APS), bromophenol blue (BPB), glycerol, $N, N, N^{\prime}, N^{\prime}$-tetramethylethylenediamine (TEMED), sodium dodecyl sulfate (SDS) and urea] and Immobiline DryStrip (pH3-10L, $24 \mathrm{~cm}$ ) were purchased from Amersham Biosciences (Uppsala, Sweden). Thiourea was purchased from Invitrogen (Carlsbad, CA, USA). Pharmalyte (pH3-10) and TRIZMA Pre-set Crystals ( $\mathrm{pH} 8.8$ ) were obtained from Sigma. HPLC grade solvents and 3-[(3-cholamidopropyl) dimethylammonio]-1-propanesulphonate (CHAPS) were purchased from Wako Pure Chemicals.

\section{Animals and treatments}

Male rats [Crj:CD(SD)IGS; 7 weeks old] were purchased from Charles-River Japan (Kanagawa, Japan). All procedures of animal experiments were approved by the Animal Ethics Committee (Internal Animal Care and Use Committee). The animals were randomly assigned to each group (control and treated group) based on their body weight. The animals were housed in individual cages, and were allowed free access to water and food (CE-7, CLEA Japan Inc., Tokyo, Japan). The room temperature was set to a range of $21-25^{\circ} \mathrm{C}$ and the light was controlled to a 12 hr light/dark cycle.

Individual rats from each treatment group $(\mathrm{N}=5)$ received a single dose of APAP, AMD, TC and CTC by oral gavage using a Teflon sonde. The dose levels were set to $1000 \mathrm{mg} / \mathrm{kg}$ for APAP, $1000 \mathrm{mg} / \mathrm{kg}$ for AMD, $2000 \mathrm{mg} / \mathrm{kg}$ for TC and $1 \mathrm{~mL} / \mathrm{kg}$ for CTC. A $0.5 \%$ methylcellulose (Metolose ${ }^{\circledR}$ SM-4000, Shin-Etsu Chemical Co., Ltd., Tokyo, Japan) containing a $0.1 \%$ tween 80 (ICN Biomedicals, Inc.) was used as vehicle and control. Twenty-four hr after the administration, 
Investigation of proteomic biomarkers in in vivo hepatotoxicity.

blood was collected from the abdominal aorta under isoflurane inhalation in order to determine the concentration of unchanged compounds in plasma and to conduct a clinical pathologic analysis. The liver was removed and then immediately frozen by immersing in liquid nitrogen, and then stored at $-80^{\circ} \mathrm{C}$ until histopathological examinations and protein extraction.

\section{Clinical pathology}

Blood for clinical pathology measurements was collected at necropsies $24 \mathrm{hr}$ after administration. Parameters of blood biochemistry such as alanine aminotransferase (ALT), aspartate aminotransferase (AST), alkaline phosphatase (ALP), $\gamma$-glutamyl transpeptidase (GGT), total bilirubin (TB), total cholesterol (T-CHOL) and triglycerides (TG) were measured using a Hitachi H-7070 automatic analyzer (Hitachi, Ltd., Tokyo, Japan).

\section{Histopathology}

The livers were fixed in $4 \%$ paraformaldehyde in $0.1 \% \mathrm{~PB}$ and embedded in paraffin. Tissue sections were stained with hematoxylin and eosin (Wako Pure Chemicals), and were examined under a light microscope.

\section{Two-dimensional gel electrophoresis (2DE) for dif- ferential protein expression}

Livers were homogenized in 2D-lysis buffer [7 M urea, $2 \mathrm{M}$ thiourea, 4\%(v/v) CHAPS, DeStreak reagent, $1 \%(\mathrm{v} / \mathrm{v})$ Pharmalyte $(\mathrm{pH} 3-10)$ and traces of $\mathrm{BPB}$ ] containing a protease inhibitor (Protease Inhibitor Mix, Amersham Biosciences) by grinding with a pestle and centrifuged at $15,000 \mathrm{~g}$ for $15 \mathrm{~min}$ at $4^{\circ} \mathrm{C}$. The supernatants of the liver from 5 animals were pooled and the protein concentration was determined with a modified Lowry's method (Lowry et al., 1951) using a RC DC Protein Assay Kit (Bio-Rad Laboratories, Hercules, CA, USA). Aliquots of the pooled liver homogenates containing $100 \mu \mathrm{g}$ of protein were loaded to immobilized $\mathrm{pH}$ gradient strip gels (linear $\mathrm{pH}$ gradient $3-10,24 \mathrm{~cm}$ ) in triplicate using sample cups at the acidic end. Isoelectric focusing was performed via step-wise voltage increments from $250 \mathrm{~V}$ to $8000 \mathrm{~V}$ until the total volt-hours reached $69 \mathrm{kV}$-hrs using an Ettan IPGphor Isoelectric Focusing Unit (Amersham Biosciences). Following two-step equilibration with 50 $\mathrm{mM}$ Tris- $\mathrm{HCl}$ (pH8.8), $6 \mathrm{M}$ urea, 39\%(v/v) glycerol, $2 \%(\mathrm{v} / \mathrm{v}) \mathrm{SDS}$ and BPB containing $1 \%(\mathrm{w} / \mathrm{v})$ DTT and $2.5 \%(\mathrm{w} / \mathrm{v})$ iodoacetamide (IAA, Sigma), the IPG strips were embedded on top of $12.5 \%$ SDS-PAGE gels (20 $\mathrm{cm} \times 26 \mathrm{~cm} \times 1 \mathrm{~mm}$ ) and sealed with $0.5 \%$ agarose. Proteins were separated based on their molecular weight. SDS-PAGE was run at the power of $20 \mathrm{~W} / \mathrm{gel}$ at $25^{\circ} \mathrm{C}$ for approximately $4 \mathrm{hr}$ using an Ettan DALTtwelve separation unit (Amersham Biosciences). 2Dgels were fixed in $10 \%(\mathrm{v} / \mathrm{v})$ ethanol and $7 \%(\mathrm{v} / \mathrm{v})$ acetic acid solution, and then stained with SyproRuby protein gel stain (Molecular Probes, Inc., Eugene, OR, USA) to visualize spots. Gel images were acquired by digital scanning at $100 \mu \mathrm{m}$ resolution using a Typhoon 9200 fluorescence image analyzer (Amersham Biosciences). Protein spots were detected, matched among groups, quantified and normalized using the Progenesis Workstation analysis system (Nonlinear Dynamics, Newcastle upon Tyne, UK). Their normalized spots volumes (percentage of total spot volume on 2D-gel) were compared between control and compound-treated groups. The criteria of differential expression were a more than 2-fold increase or decrease of the normalized spot volumes.

\section{In-gel tryptic digestion}

Protein spots of interest were excised using a ProXcision proteomics gel-cutting robot (PerkinElmer, Boston, MA, USA) from the preparative gels, which were separated the aliquots of liver homogenate containing $500 \mu \mathrm{g}$ protein. Proteins in the gel plugs were reduced in $10 \mathrm{mM}$ DTT at $56^{\circ} \mathrm{C}$ and then alkylated in $25 \mathrm{mM}$ IAA at $45^{\circ} \mathrm{C}$ and then washed in $25 \mathrm{mM}$ ammonium bicarbonate. Gel plugs were then dehydrated in acetonitrile and then dried in a vacuum centrifuge (SPD2010 Speed-Vac, ThermoSavant, Waltham, MA, USA). They were then rehydrated in $20 \mathrm{ng} / \mathrm{mL}$ of TPCK-modified trypsin (Promega, Madison, WI, USA) at $4^{\circ} \mathrm{C}$. In-gel tryptic digestion was carried out overnight at $37^{\circ} \mathrm{C}$. The digested peptides were recovered into $5 \%(\mathrm{v} / \mathrm{v})$ aqueous formic acid (Wako Pure Chemicals).

\section{Proteins identification}

Tryptic peptides were subjected to peptide sequencing tags (PSTs) for protein identification using MS/MS spectra derived from capillary liquid chromatography coupled to a hybrid quadrupole orthogonal acceleration time-of-flight mass spectrometer (CapLC and Q-TOF Ultima API, Micromass, Manchester, UK) equipped with a nano-ESI ion source. Peptides injected to the liquid chromatograph were first trapped and desalted isocratically on an LC-Packings PepMap $\mathrm{C}_{18}$ $\mu$-precolumn cartridge $(5 \mu \mathrm{m}, 100 \AA, 300 \mu \mathrm{m}$ id $\times 5$ $\mathrm{mm}$, Dionex, San Francisco, CA, USA) and then sepa- 
rated on an analytical PepMap $\mathrm{C}_{18}$ capillary column (3 $\mu \mathrm{m}, 100 \AA, 75 \mu \mathrm{m}$ id $\times 15 \mathrm{~cm}$, Dionex) at $200 \mathrm{~nL} / \mathrm{min}$ using a $60 \mathrm{~min}$ isocratic gradient of $5-80 \%$ acetonitrile containing $0.1 \%$ formic acid. Product ion spectra were automatically acquired by data-dependent exact product ion analysis at the criteria of intensity and charges $(+2$ and +3$)$ of precursor ions. The datasets of the MS/ MS spectra, including peptides sequence information, were searched against the SWISS-PROT (GeneBio, Geneva, Switzerland) and NCBInr database using Mascot Daemon (Matrix Science, London, UK) as a client attached to the Mascot search protocol.

\section{Statistical analysis}

Dunnett's multiple comparison procedure ( $\mathrm{Gad}$ and Weil, 1994) was used for statistical analyses of clinical pathology parameters. Protein expression data are expressed as the mean \pm SD of 3 replicate gels for fold-changes of normalized spot volumes. For the statistical analysis of these data, Student's $t$-test (Gad and Weil, 1994) was used, and $p<0.05$ was set as the level of statistical significance. Agglomerative hierarchical clustering analysis using Ward's algorithm (Ward, 1963) was performed by a JMP ver. 5.1 software package (SAS Institute Inc., Cary, NC, USA).

\section{RESULTS AND DISCUSSION}

\section{Blood biochemistry and histopathology}

Treatment-related changes in parameters of blood biochemistry are summarized in Table 1. Increased ALT, AST and GGT activities were detected in rats treated with APAP and CTC. Lipid metabolismassociated parameters, such as T-CHOL and TG, were altered by the administrations of AMD, TC and CTC. Although enormous variations in ALT and AST values were observed in the APAP-treated group, all rats were applied to a proteomic analysis by the judgment from histopathological findings.

Histopathological findings are summarized in Table 2. Inflammatory changes were observed in all treatment groups. In addition, necrotic cells and eosinophilic changes were detected in rats treated with APAP and CTC, and fat deposits were found in those treated with TC and CTC.

These findings showed a consequence of liver damage and correlated well with the mechanisms of hepatotoxicity of the tested compounds, and the applicable liver would be sufficient to estimate the proteomic changes.

Table 1. Treatment-related changes in blood chemistry induced by APAP, AMD, TC and CTC.

\begin{tabular}{cccccccccc}
\hline & \multicolumn{2}{c}{ Control } & \multicolumn{2}{c}{ APAP } & \multicolumn{2}{c}{ AMD } & TC & CTC \\
\hline \hline ALT (U/L) & $41.8 \pm$ & \pm .77 & $209.0 \pm 252.3$ & $42.2 \pm$ & 4.09 & $36.6 \pm 1.52^{*}$ & $82.0 \pm 22.8^{*}$ \\
AST (U/L) & $73.6 \pm$ & \pm .20 & $573.3 \pm 490.1$ & $70.2 \pm$ & \pm 3.03 & $67.0 \pm 6.93$ & $131.8 \pm 21.25^{*}$ \\
ALP (U/L) & $934.8 \pm 196.3$ & $1229.8 \pm 293.62$ & $1454.0 \pm 236.40^{*}$ & $788.8 \pm 59.76$ & $918.8 \pm 139.9$ \\
GGT (U/L) & $0.0 \pm$ & \pm .0 & $0.5 \pm$ & \pm .6 & $0.2 \pm$ & \pm .5 & $0.0 \pm 0.0$ & $0.4 \pm$ & 0.6 \\
TB (mg/dL) & $0.10 \pm$ & 0.00 & $0.13 \pm$ & 0.05 & $0.10 \pm$ & 0.00 & $0.10 \pm 0.00$ & $0.10 \pm 0.00$ \\
T-CHOL (mg/dL) & $71.2 \pm$ & 9.04 & $85.8 \pm$ & $\pm .85^{*}$ & $107.6 \pm 14.99 *$ & $59.8 \pm 6.34$ & $60.0 \pm 13.0$ \\
TG (mg/dL) & $119.8 \pm$ & \pm 4.19 & $109.0 \pm 51.47$ & $29.2 \pm$ & $\pm .09^{*}$ & $58.2 \pm 28.2^{*}$ & $46.8 \pm 16.6^{*}$ \\
\hline
\end{tabular}

Data represent mean \pm SD of 5 animals except for APAP-treated group $(n=4)$.

ALT: alanine aminotransferase, AST: aspartate aminotransferase, ALP: alkaline phosphatase, GGT: $\gamma$-glutamyl transpeptidase, TB: total bilirubin, T-CHOL: total cholesterol, TG: triglyceride

*: statistically significant difference from the control group $(\mathrm{p}<0.05)$.

Table 2. Histopathological findings of male rat liver $24 \mathrm{hr}$ after administration of APAP, AMD, TC and CTC.

\begin{tabular}{|c|c|c|c|c|}
\hline & APAP & AMD & $\mathrm{TC}$ & CTC \\
\hline Inflammatory cell infiltration & + & \pm & $\pm \sim+$ & ++ \\
\hline Eosinophilic changes of hepatocytes & + & - & - & + \\
\hline Necrosis of hepatocytes & ++ & - & - & ++ \\
\hline Fatty changes of hepatocytes & - & - & + & ++ \\
\hline
\end{tabular}

Grade of changes; -: NAD, \pm : Slight, +: Mild, ++: Severe. 
Investigation of proteomic biomarkers in in vivo hepatotoxicity.

\section{Effects of hepatotoxicants on protein expression in the liver}

Total rat liver proteins solubilized in lysis buffer containing $7 \mathrm{M}$ urea and $2 \mathrm{M}$ thiourea were clearly separated into over 2,000 spots on 2D-gels. Differentially expressed spots showing more than 2 -fold changes from their normalized spot volume were 37, 28, 36 and 94 by the treatments with APAP, AMD, TC and CTC, respectively (Table 3 ). Following protein identifications based on the spots and MS/MS data, these affected proteins were categorized according to their biological functions as referred to gene ontology (Table 4). It was found that proteins related to the metabolism of amino acids, carbohydrates, fatty acids and lipids, and stress responses were mainly affected. APAP induced remarkable changes in the expression of necrotic cell death-related proteins such as arginase, catalase, heat shock $70 \mathrm{kDa}$ protein (HSP70), regucalcin (senescence marker protein-30), and superoxide disumutase (Mn-SOD). The involved proteins to process of lipid and fatty acid metabolism, such as HMGCoA synthase, were affected by the treatments with AMD and TC. In addition, AMD and CTC treatments affected the expression of acyl-CoA dehydrogenase and 2-oxovalerate dehydrogenase, which were known to contribute to the $\beta$-oxidation pathway of lipids. Their changes were well correlated with the previous finding that drug-induced liver steatosis could result from perturbation of the $\beta$-oxidation pathway (Berson et al., 1998; Letteron et al., 1996). In the CTC treatment group, the number of proteins showing an altered expression was larger than that found in the other groups (Table 3 ). From the clinical pathological and histopathological point of view, the liver of rats administered CTC was considered to be in more severe condition compared to the other 3 groups; thus, these changes in protein expression were well correlated with the macroscopical changes observed.

\section{Selection of candidate proteins showing differential expression}

Although the mechanism of hepatotoxicity differed among the hepatotoxic compounds, it was demonstrated that 8 proteins were affected by all four toxicants examined (Fig. 1). Some of them were identified by Q-Tof mass spectrometry as up-regulated proteins; carbonic anhydrase III (P14141), 60kDa heat shock protein (P63039), glutamate dehydrogenase (GDH, P10860), adenylate kinase isoenzyme 4 (Q9WUS0), NADP-dependent malic enzyme (MAOX, P13697) and 2-oxoisovalerate dehydrogenase $\alpha$ subunit (P11960). Serotransferrin (TRFE, P12346) and NDRG1 related protein NDRG2b1 (NDRG2, gi17977874) were down-regulated (Fig. 2).

Carbonic anhydrase III (CA3) is a cytoplasmic enzyme that is abundant in the liver as well as in skeletal muscle, and plays a role in the cellular response to oxidative stress. It was reported that the overexpression of CA3 protects cells from oxidative stress by reducing intracellular ROS (Raisanen et al., 1999; Kim et al., 2004). The $60 \mathrm{kDa}$ heat shock protein (HSP60) is one of the molecular chaperone proteins, which participate in protein folding, unfolding, and translocation across membranes in organelles such as mitochondria (Craig et al., 1993). Needless to say, HSP60 is also expressed in response to stress. HSP60 in the liver was reported to be unaffected or down-regulated by APAP in mice (Salminen et al., 1997a; Ruepp et al., 2002). Salminen et al. (1997b) also documented that different HSPs were affected in rat versus mouse liver. In fact, Huang et al. (2002) and Meneses-Lorente et al. (2004) demonstrated in proteomics studies that HSP60 protein was up-regulated in PC12 cells undergoing lipopolysaccharide-induced apoptosis and in rat liver showing druginduced steatosis, respectively. These events were consistent with our observations on HSP60 in rat liver. Adenylate kinase 4 (AK4), which is also called ATPAMP transphosphorylase, is a relatively newly identi-

Table 3. The number of differentially expressed spots of rat liver proteins on $2 \mathrm{D}$-gels at $24 \mathrm{hr}$ after treatment with a single oral dosing of APAP, AMD, TC and CTC.

\begin{tabular}{cccc}
\hline & Up-regulated & Down-regulated & Total \\
\hline \hline APAP & 20 & 17 & 37 \\
AMD & 16 & 12 & 28 \\
TC & 23 & 13 & 36 \\
CTC & 57 & 36 & 94 \\
\hline
\end{tabular}

Criteria for differential expression: $\geq 2$-fold changes and $\mathrm{p}<0.05$. 
T. YAMAMOTO et al.

Table 4. Major functional categories of proteins induced and reduced by treatments with hepatotoxicants in rat liver.

\begin{tabular}{ll}
\multicolumn{1}{c}{ APAP } & \multicolumn{1}{c}{ AMD } \\
\hline \hline & \multicolumn{1}{c}{ Amino Acid } \\
$\begin{array}{ll}\text { - 2-oxoisovalerate dehydrogenase } \alpha \\
\text { subunit }\end{array}$ & $\begin{array}{l}\text {-2-oxoisovalerate dehydrogenase } \alpha \\
\text { subunit }\end{array}$ \\
- Arginase 1 & $\bullet$ C-1-tetrahydrofolate synthase \\
- Glutamate dehydrogenase 1 & - Dimethylglycine dehydrogenase \\
- Peptide methionine sulfoxide reductase & - Glutamate dehydrogenase 1 \\
- Phenylalanine-4-hydroxylase & - Insulin-degrading enzyme \\
& - Propionyl-CoA carboxylase $\beta$ chain
\end{tabular}

Metabolism
-2-oxoisovalerate dehydrogenase $\alpha$
subunit
- C-1-tetrahydrofolate synthase
- C-1-tetrahydrofolate synthase
- Glutamate dehydrogenase 1
- Propionyl-CoA carboxylase $\beta$ chain

- 2-oxoisovalerate dehydrogenase $\alpha$ subunit

- 3-hydroxyisobutyrate dehydrogenase

- Aspartate aminotransferase

- Dihydropteridine reductase

- Glutamate dehydrogenase 1

- Phenylalanine-4-hydroxylase

- Propionyl-CoA carboxylase $\beta$ chain

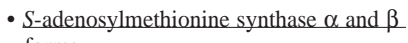
forms

Carbohydrate Metabolism /Glucose Metabolism / Alcohol Metabolism / Energy Metabolism

- Adenylate kinase isoenzyme 4

- Alcohol dehydrogenase [NADP+]

- Estrogen sulfotransferase, isoform

- Fructose-bisphosphate aldolase B

- Fructose-bisphosphate aldolase B

- Translational endoplasmic reticulum ATPase
- Adenylate kinase isoenzyme 4

- Aldehyde dehydrogenase

- ATP synthase $\alpha$ chain

- Fructose-bisphosphate aldolase B
- Adenylate kinase isoenzyme 4 - $\alpha$-enolase

- Pyruvate kinase isozymes R/L

- Adenylate kinase isoenzyme 4

- Alcohol dehydrogenase class III

- Aldehyde dehydrogenase

- ATP synthase $\alpha$ chain

- ATP synthase $\beta$ chain

- ATP synthase $\gamma$ chain

- Electron transfer flavoproteinubiquinone oxidoreductase

- Fructose-bisphosphate aldolase B

- Glycogen phosphorylase, liver form

- Ketohexokinase

- Pyruvate kinase isozymes R/L

- Sorbitol dehydrogenase

\section{Fatty Acid Metabolism}

- Acyl-CoA dehydrogenase, long-chain specific

- Long-chain-fatty-acid-CoA ligase 1
- Acyl-CoA dehydrogenase, long-chain
specific Short chain 3-hydroxyacyl-CoA dehydrogenase

$$
\begin{aligned}
& \text { - Acyl-CoA } \\
& \text { specific }
\end{aligned}
$$

- Acyl-CoA dehydrogenase, long-chain specific

- Long-chain-fatty-acid-CoA ligase

- Short chain 3-hydroxyacyl-CoA dehydrogenase

\section{Lipid Metabolism / Cholesterol Metabolism}

- Fatty acid-binding protein

- NADP-dependent malic enzyme

- Hydroxymethylglutaryl-CoA synthase

- Hydroxymethylglutaryl-CoA synthase

- Hydroxymethylglutaryl-CoA synthase

- Hydroxymethylglutaryl-CoA synthase

- Similar to microsomal triglyceride transfer protein

- NADP-dependent malic enzyme

- NADP-dependent malic enzyme

- Triosephosphate isomerase

\section{• Aflatoxin Bl aldehyde reductase member \\ - Aflatoxin B1 aldehyde reductase member 1 \\ - Glutathione $S$-transferase 8 \\ - Glutathione $S$-transferase Ya1 \\ - Glutathione $S$-transferase Ya-2 \\ - $\underline{\text { N-hydroxyarylamine sulfotransferase }}$}

- $\alpha$-1-antiproteinase precursor

- Uroporphyrinogen decarboxylase
Xenobiotic Metabolism / GSH Metabolism / Detoxification

- Aryl sulfotransferase

- Glutathione $S$-transferase Yb-1

- Glutathione $S$-transferase Ya-2

- 3- $\alpha$-hydroxysteroid dehydrogenase

- Dihydrolipoyllysine-residue acetyltransferase component of pyruvate dehydrogenase complex

- Hydroxyacid oxidase 3

- Hydroxymethylglutaryl-CoA lyase

- Hydroxymethylglutaryl-CoA synthase

- NADP-dependent malic enzyme

- Triosephosphate isomerase

- Cytochrome P450 2C11

- N-hydroxyarylamine sulfotransferase

- Glutathione peroxidase

- Glutathione S-transferase 8

- Glutathione S-transferase Ya-2

- Glutathione S-transferase Yb-3

Protein Degradation / Protein Biosynthesis / Heme Biosynthesis

- 26S protease regulatory subunit 8

- 26S protease regulatory subunit 8

- Ubiquitin-conjugating enzyme E2 D3

- $\delta$-aminolevulinic acid dehydrogenase

- 40 S ribosomal protein SA

- Proteasome subunit $\alpha$ type 2

- Proteasome subunit $\alpha$ type 6

- Ubiquitin-conjugating enzyme E2 D3

- $\delta$-aminolevulinic acid dehydrogenase

RNA processing /Nucleic Acid Metabolism

- Nucleic acid binding factor pRM10 •

- Heterogeneous nuclear ribonucleoprotein A3 - Heterogeneous nuclear ribonucleoprotein $\mathrm{K}$

- Nucleic acid binding factor pRM10

- Adenylyl cyclase-associated protein 1

- Deoxyribonuclease II $\beta$ 
Investigation of proteomic biomarkers in in vivo hepatotoxicity.

Table 4. Continued.

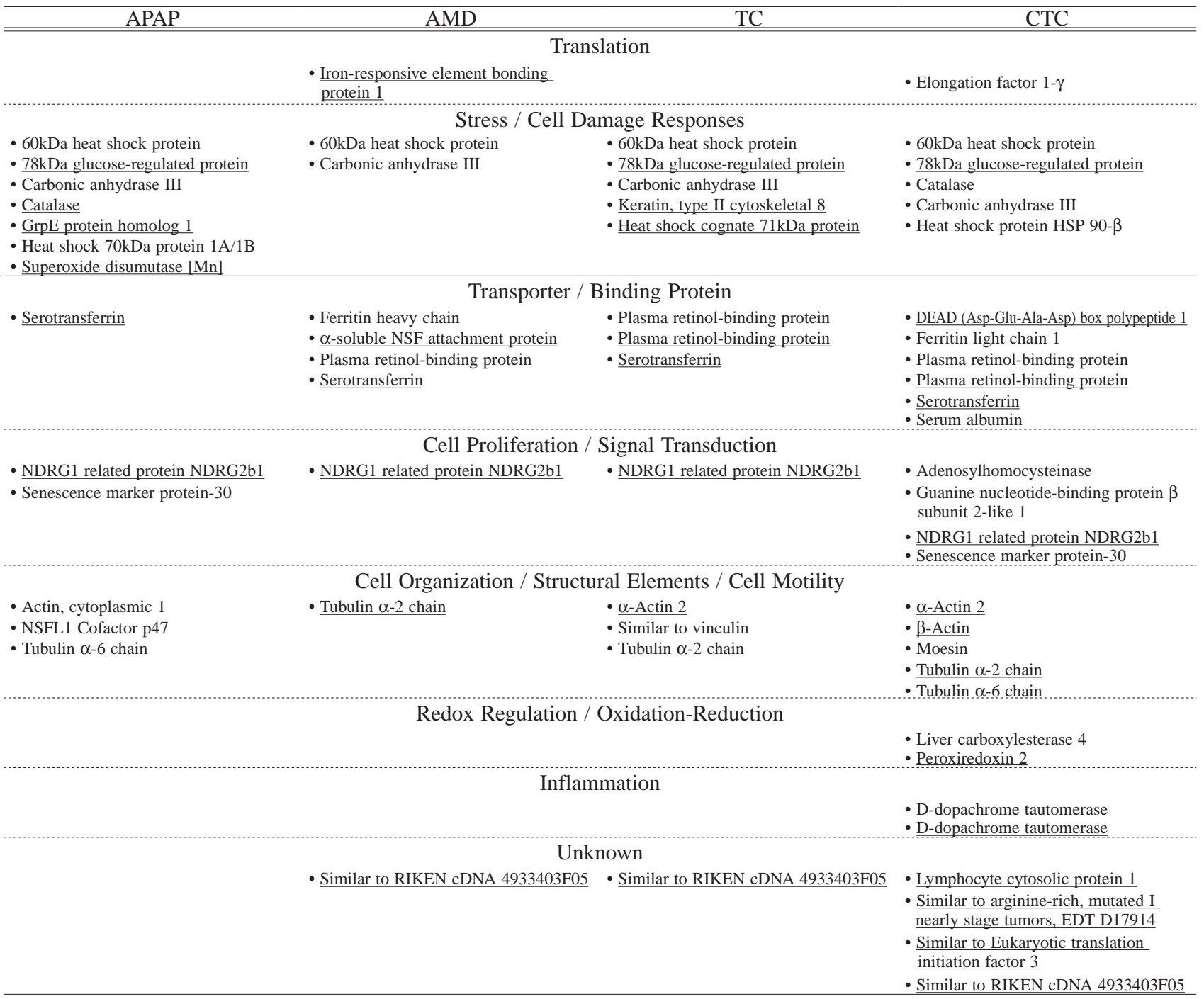

Only successfully identified proteins with $\geq 2$-fold expression change and $\mathrm{p}<0.05$ are included in the table.

Underline indicates down-regulated protein.

fied 4th enzyme of the adenylate kinase family, and its expression is restricted to the brain and liver, while the other isozymes are ubiquitously expressed (Yoneda $e t$ al., 1998). AK4 is considered to act specifically on the energy metabolism rather than in the control of the homeostasis of adenine nucleotide which the other isozymes act on, but the function of AK4 has not been well characterized yet. A different subtype of adenylate kinase, $\mathrm{AK} 2$, was reported to be released concomitantly with cytochrome $c$, which is known as an apoptosis-related factor (Kohler et al., 1999). 2-Oxoisovalerate dehydrogenase (ODBA), another protein found to be up-regulated in this study, is known to be involved in the metabolism of 2-oxo acids derived from branched chain amino acids (Massey et al., 1976), and this enzyme in the liver was reported to be induced with a sign of steatosis (Meneses-Lorente et al., 2004).

We have already reported the decreased expression of glutathione peroxidase (GPX1), an oxidative stress-related protein, in rat hepatocytes exposed to APAP, AMD and TC (Yamamoto et al., 2005). The spots corresponding to GPX1 on 2D-gels in vivo did not show a more than 2 -fold alteration in rat liver. Missing in detection of the same oxidative stressrelated proteins in both in vivo and in vitro might be attributed to the repair by redox pathway-related pro- 
T. YAMAMOTO et al.

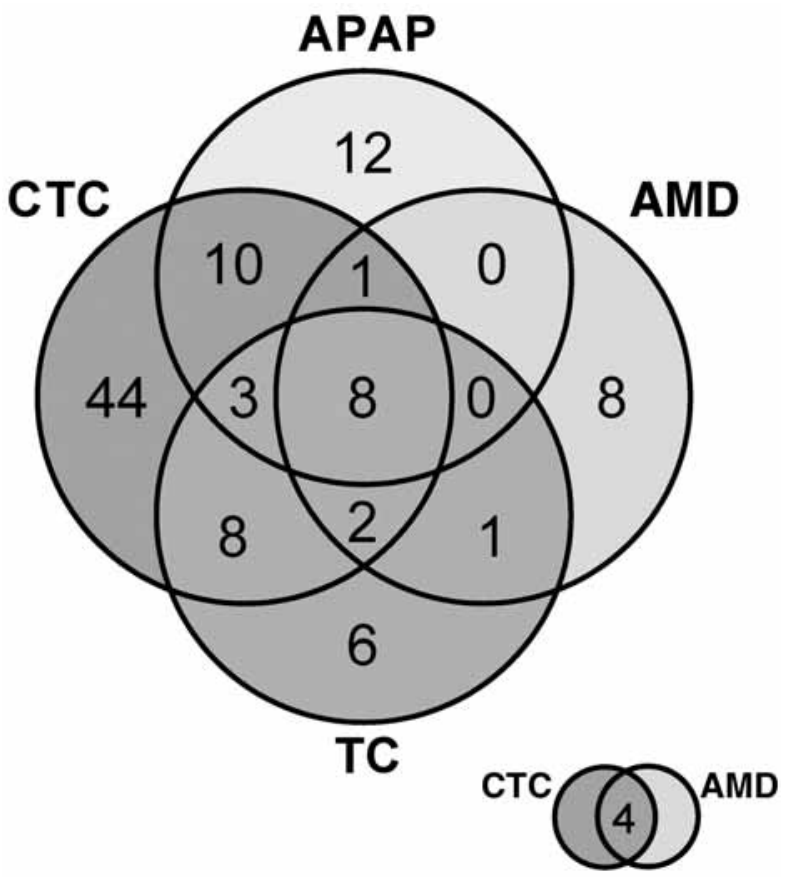

Fig. 1. Venn diagram showing the number of differentially expressed spots of the treatments with APAP, AMD, TC and CTC. This figure showed that 8 proteins were affected by all 4 compounds examined. teins, different susceptibility to oxidative stress and the participation of other pathways. In fact, the decreases in antioxidative proteins including GPX1 in the liver were detected in mice and rats around $1 \mathrm{hr}$ after administration of APAP in vivo, and then their expression levels were recovered at $24 \mathrm{hr}$ post-dosing (Arnaiz et al., 1995; Chen and Lin, 1996; Noriega et al., 2000; O’Brien et al., 2000).

\section{Differentiation of the stage of hepatotoxicity based on pattern recognition analysis}

To classify compound-treated groups into several informative groups based on the level of toxicity, the usage of protein expression information on 2D-gels was evaluated. Multivariate statistical analysis, such as hierarchical clustering, is one of the most popular methods to analyze large-scale gene expression data (Eisen et al., 1998; Waring et al., 2001). Hence there have been few applications using these kinds of analyses in the field of protein expression. We applied a twodimensional hierarchical clustering analysis to the changes of normalized spot volumes on 2D-gels. This analysis facilitated the visualization of groupings based on protein expression changes, potentially reflecting the underlying molecular mechanism of tox-

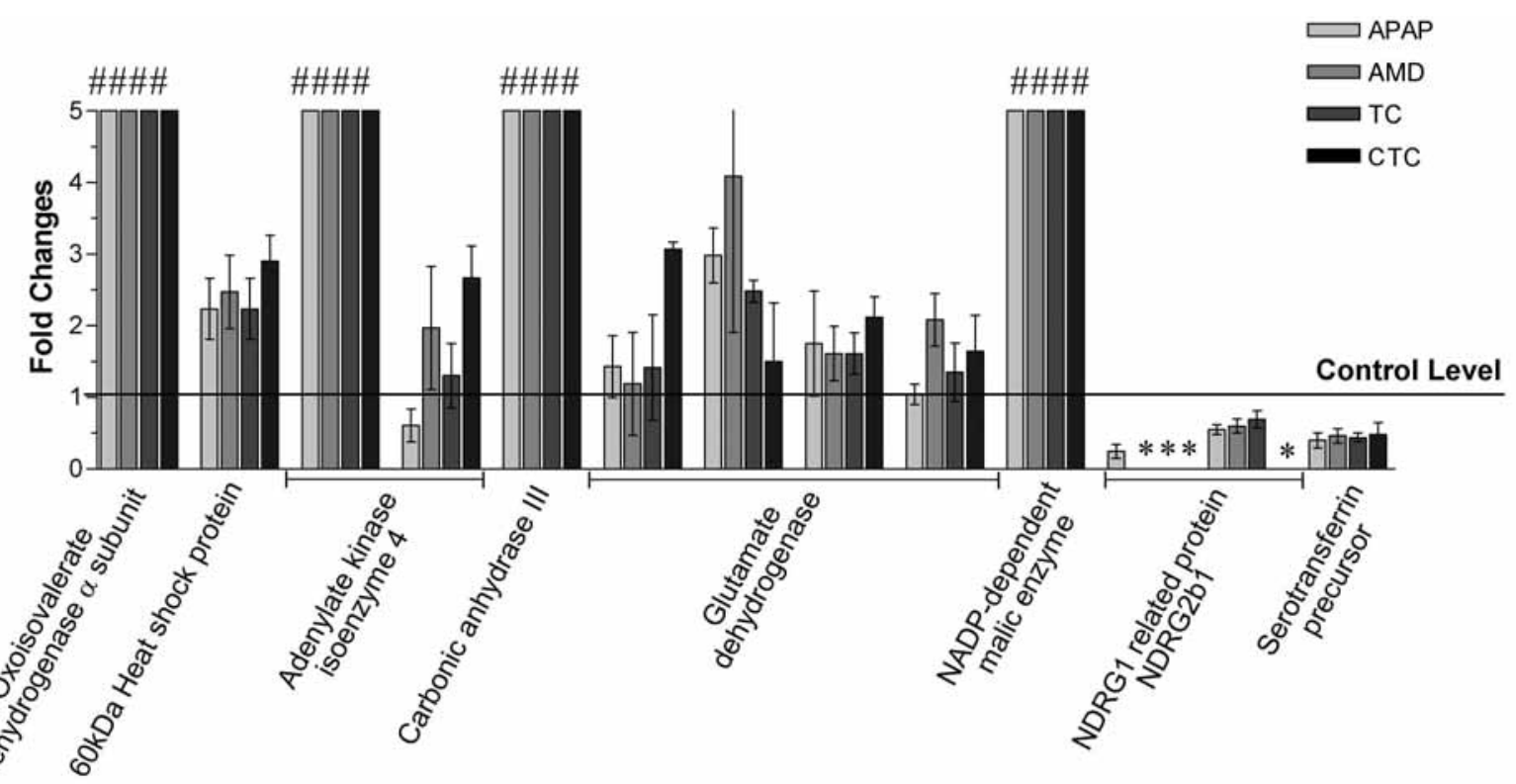

Fig. 2. Changes in the expression of the 8 proteins that were affected by the four hepatotoxicants tested. Values are expressed as foldchanges of the control values, indicated by the horizontal control level line. Data represent mean \pm SD of 3 assays.

\#: unique protein detected in the treated gels because it was not detected in the control gels and/or was under the limit of deection.

*: unique protein detected in the control gels because it was not detected in the treated gels and/or was under the limit of deection. Changes in the expression of adenylate kinase isoenzyme 4, glutamate dehydrogenase and NDRG1 related protein NDRG2b1 were detected on more than one spot on 2D gels. 
Investigation of proteomic biomarkers in in vivo hepatotoxicity.

icity and severity of liver damage. A hierarchical clustering map generated with all protein spots detected on the 2D-gels is shown in Photo 1 (A) with the severity of liver damage. This analysis formed two major clusters.
One is the cluster including CTC and APAP, and the other is that including control, AMD and TC. Regarding treatment with CTC, the most severe condition would be expected because of the largest number of
(A)
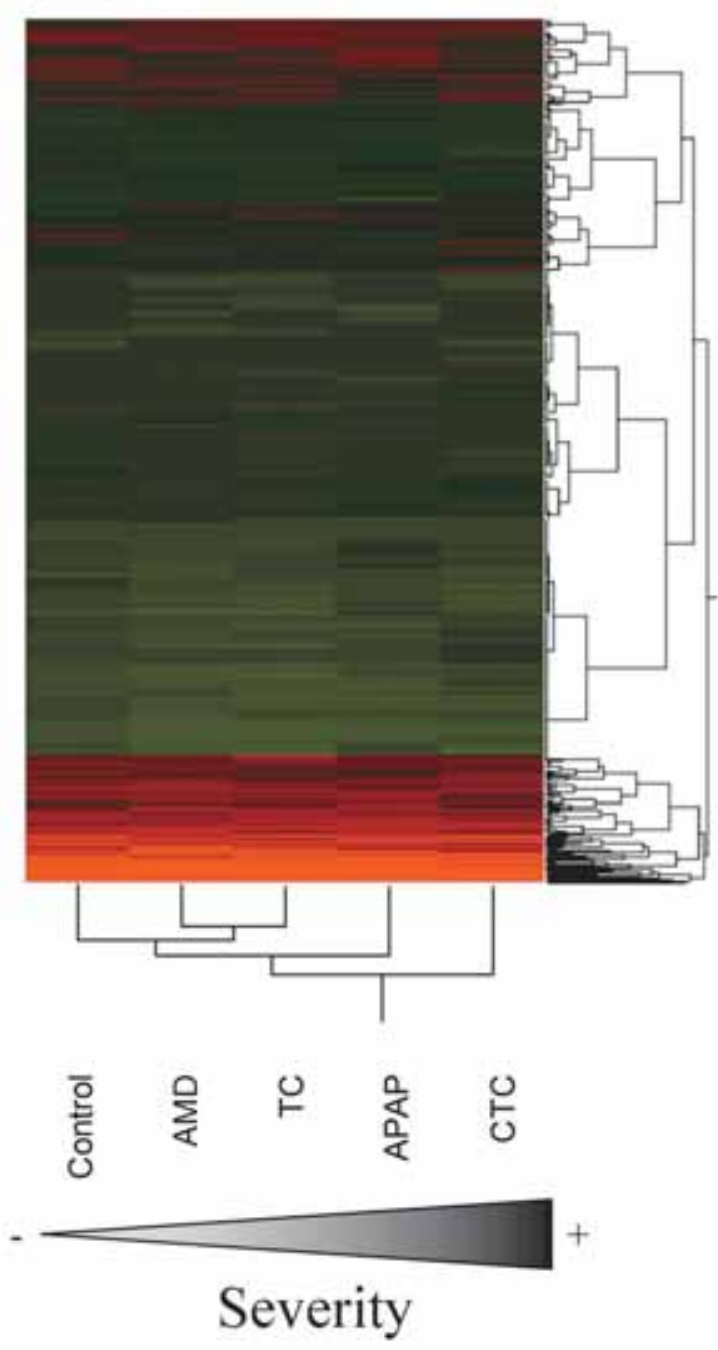

(B)
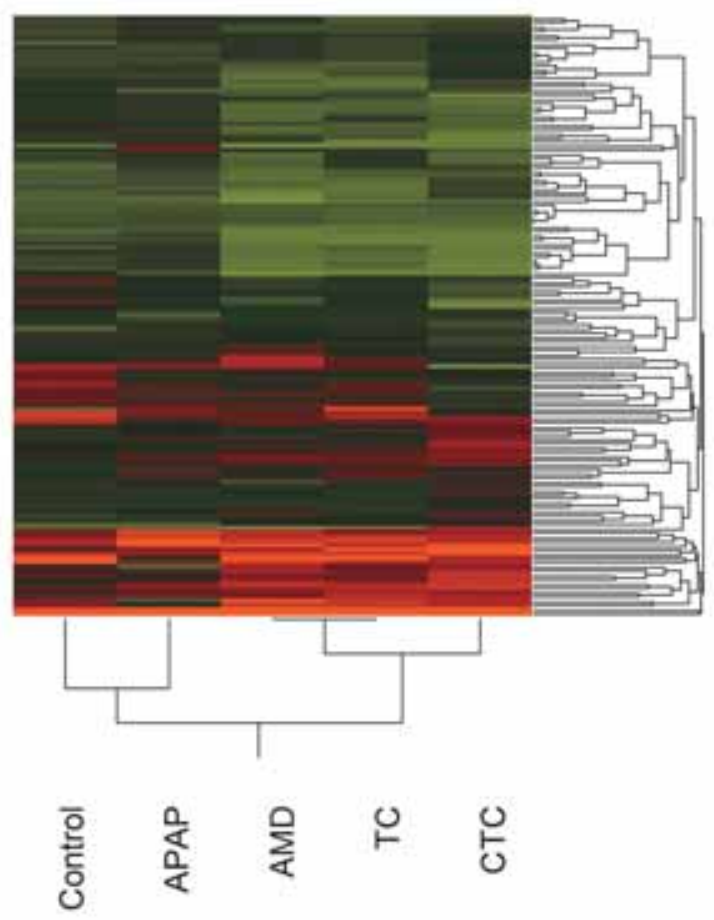

(C)

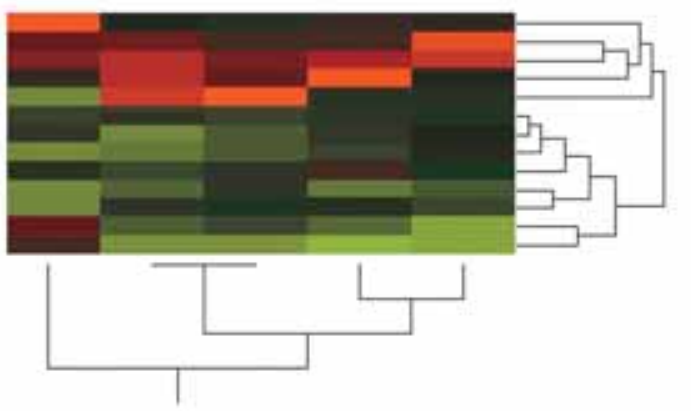

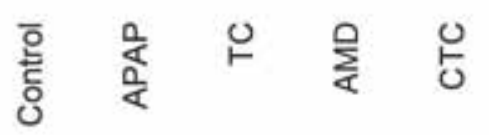

Photo 1. Two-dimensional hierarchical clustering map using protein expression patterns induced by APAP, AMD, TC and CTC in male rats. Clusters were generated with normalized volumes of all spots detected (A), all significant differentially expressed spots (B) and 8 differentially expressed proteins (C) on matched 2D-gel images. Map (A) represents the severity of hepatotoxicity derived from histopathology and blood chemistry. 
altered proteins and the observed histopathological changes. APAP-caused histopathological changes and elevated liver enzymes revealed this was the second severe condition given. On the other hand, slight changes were observed in histopathology and blood biochemistry with AMD and TC. Thus, their effects were milder than those observed in the CTC and APAP groups although several changes in protein expression were detected. Therefore, this hierarchical clustering map seemed to correlate with the observations of conventional histopathological and clinical pathological findings of hepatotoxicity. Moreover, hierarchical clustering was carried out for all significant differentially expressed spots [Photo 1, (B)], and it was not possible to clearly differentiate them into meaningful groups. Finally, the only differentially expressed spots in common, corresponding to the 8 identified proteins, were used to generate clusters [Photo 1 (C)]. Photo 1 (C) showed the expression patterns grouped into two clusters corresponding to non-toxic control and toxic treated groups (APAP, AMD, TC and CTC). These results suggested that this hierarchical clustering analysis using spot information on 2D-gels was useful to screen animals based on the effects on protein expression.

In conclusion, we could identify 8 liver proteins CA3, HSP60, GDH, AK4, MAOX, ODBA, TRFE and NDRG2 - which were affected by all the 4 hepatotoxicants. Among them, proteins related to the response to an oxidative stress, the metabolism of energy and lipids, and $\beta$-oxidation of fatty acids were found. Monitoring one or more of these proteins may provide important information on the effect of compounds on the liver. Moreover, in the present study, it was demonstrated that pattern recognition analysis such as hierarchical clustering using protein expression patterns, which was derived from 2D-gels, correlated well with conventional toxicological endpoints such as findings from clinical pathology and histopathology.

We would like to emphasize that there is a fair possibility of toxicological evaluation using comprehensive protein expression profiles in the liver. Although further studies using the liver from individual animals are needed to obtain a more precise link with phenotype, this kind of multivariate statistical analysis using proteins expression pattern may become widely applicable for early screening of toxicity.

\section{REFERENCES}

Arnaiz, S.L., Llesuy, S., Curtrin, J. and Boveris, A.
(1995): Oxidative stress by acute acetaminophen administration in mouse liver. Free Radic. Biol. Med., 19, 303-310.

Bandara, L.R. and Kennedy, S. (2002): Toxicoproteomics - A new preclinical tool. Drug Discov. Today, 7, 411-418.

Berson, A., De Beco, V., Letteron, P., Robin, M.A., Moreau, C., El Kahwaji, J., Verthier, N., Feldmann, G., Fromentry, B. and Pessayre, D. (1998): Steatohepatitis-induced drugs cause mitochondrial dysfunction and lipid peroxidation in rat hepatocytes. Gastroenterology, 114, 764-774.

Chen, L.H. and Lin, S.M. (1996): Acetaminophen alters antioxidant defense enzyme activities in hepatocyte culture. Biochem. Arch., 12, 33-42.

Chitturi, S. and George, J. (2002): Hepatotoxicity of commonly used drugs: Nonsteroidal antiinflammatory drugs, antihypertensives, antidiabetic agents, anticonvulsants, lipid-lowering agents, psychotropic drugs. Semin. Liver Dis., 22, 169-183.

Craig, E.A., Gambill, B.B. and Nelson, R.J. (1993): Heat shock proteins: Molecular chaperones of protein biogenesis. Microbiol. Rev., 57, 402414.

Deboyser, D., Goethals, F., Krack, G. and Roberfroid, M. (1989): Investigation into the mechanism of tetracycline-induced steatosis: Study in isolated hepatocytes. Toxicol. Appl. Pharmacol., 97, 473-479.

Eisen, M.B., Spellman, P.T., Brown, P.O. and Botstein, D. (1998): Cluster analysis and display of genome-wide expression patterns. Proc. Natl. Acad. USA., 95, 14863-14868.

Faich, G.A. and Moselry, R.H. (2001): Troglitazone (Rezulin) and hepatic injury. Pharmacoepidemiol. Drug Safety, 10, 537-547.

Gad, S.C. and Weil, C.S. (1994): Statistics for toxicologists. In Principles and Methods of Toxicology (Hayes, A.W., ed.), 3rd ed., pp. 221-274, Raven Press, New York.

Gitlin, N., Julie, N.L., Spurr, C.L., Lim, K.N. and Juarbe, H.M. (1998): Two cases of severe clinical and histologic hepatotoxicity associated with troglitazone. Ann. Intern. Med., 129, 36-38.

Goldman, I.S., Winkler, M.L., Raper, S.E., Baker, M.E., Keung, E., Goldberg, H.I. and Boyer, T.D. (1985): Increased hepatic density and phospholipidosis due to amiodarone. Am. J. Roentol., 144, 541-546. 
Investigation of proteomic biomarkers in in vivo hepatotoxicity.

Hayes, K.R. and Bradfield, C.A. (2005): Advances in toxicogenomics. Chem. Res. Toxicol., 18, 403414.

Henry, C.J. (2003): Evolution of toxicology for risk assessment. Int. J. Toxicol., 22, 3-7.

Herrine, S.K. and Choudhary, C. (1999): Severe hepatotoxicity associated with troglitazone. Ann. Intern. Med., 130, 163-164.

Huang, Y.H., Chang, A.Y.W., Huang, C.M., Huang, S.W. and Chan, S.H.H. (2002): Proteomic analysis of lipopolysaccharide-induced apoptosis in PC12 cells. Proteomics, 2, 1220-1228.

Hunter, E.B., Johnston, P.E., Tanner, G., Pinson, C.W. and Awad, J.A.(1999): Bromfenac (Duract)associated hepatic failure requiring liver transplantation. Am. J. Gastroenterol., 94, 22992301.

Kennedy, S. (2002): The role of proteomics in toxicology: Identification of biomarkers of toxicity by protein expression analysis. Biomarkers, 7, 269290.

Kim, G., Lee, T.H., Wetzel, P., Geers, C., Robinson, M.A., Myers, T.G., Owens, J.W., Wehr, N.B., Eckhaus, W., Gros, G., Wynshaw-Boris, A. and Levine, R. (2004): Carbonic anhydrase III is not required in the mouse for normal growth, development, and life span. Mol. Cell. Biol., 24, 9942-9947.

Kohler, C., Gahm, A., Noma, T., Nakazawa, A., Orrenius, S. and Zhivotovsky, B. (1999): Release of adenylate kinase 2 from the mitochondrial intermembrane space during apoptosis. FEBS Lett., 447, 10-12.

Letteron, P., Brahimi-Bourouina, N., Robin, M.A., Moreau, A., Feldmann, G. and Pessayre, D. (1996): Glucocorticoids inhibit mitochondrial matrix acyl-CoA dehydrogenases and fatty acid beta-oxidation. Am. J. Physiol. Gastrointest. Liver Physiol., 272, G1141-G1150.

Lowry, O.H., Rosebrough, N.J., Farr, A.L. and Randall, R.J. (1951): Protein measurement with the folin phenol reagent. J. Biol. Chem., 193, 265-275.

MacGregor, J.T. (2003): The future of regulatory toxicology: Impact of the biotechnology revolution. Toxicol. Sci., 75, 236-248.

Maddrey, W.C. (2005): Drug-induced hepatotoxicity. J. Clin. Gastroenterol., 39, S83-S89.

Massey, L.K., Sokatch, J.R. and Conrad, R.S. (1976): Branched-chain amino acid catabolism in bacteria. Bacteriol. Rev., 40, 42-54.
Meneses-Lorente, G., Guest, P.C., Lawrence, J., Muniappa, N., Knowles, M.R., Skynner, H.A., Salim, K., Cristea, I., Mortishire-Smith, R., Gaskel, S.J. and Watt, A. (2004): A proteomic investigation of drug-induced steatosis in rat liver. Chem. Res. Toxicol., 17, 605-612.

Moses, P.L., Schroeder, B., Alkhatib, O., Ferrentino, N., Suppan, T. and Lidofsky, S.D. (1999): Severe hepatotoxicity associated with bromfenac sodium. Am. J. Gastroenterol., 94, 1393-1396.

Nelson, S.D. (1990): Molecular mechanisms of the hepatotoxicity caused by acetaminophen. Semin. Liver Dis., 10, 267-278.

Nicholson, J.K., Lindon, J.C. and Holmesm, E. (1999): 'Metabonomics': Understanding the metabolic responses of living systems to pathophysiological stimuli via multivariate statistical analysis of biological NMR spectroscopic data. Xenobiotica, 29, 1181-1189.

Noriega, G.O., Ossola, J.O., Tomaro, M.L. and Batlle, A.M. (2000): Effect of acetaminophen on heme metabolism in rat liver. Int. J. Biochem. Cell Biol., 32, 938-991.

O'Brien, P.J., Slaughter, M.R., Swain, A., Birmingham, J.M., Greenhill, R.W., Elcock, F. and Bugelski, P.J. (2000): Repeated acetaminophen dosing in rats: Adaptation of hepatic antioxidant system. Human Exp. Toxicol., 19, 277-283.

Pennington, S.R. and Dunn, M.J. (2001): Proteomics from protein sequence to function. SpringerVerlag, New York.

Poucell, S., Ireton, J., Valencia-Mayoral, P., Downar, E., Larratt, L., Patterson, J., Blendis, L. and Phillips, M.J. (1984): Amiodarone-associated phospholipidosis and fibrosis of the liver: Light, immunohistochemical, and electron microscopic studies. Gastroenterology, 86, 926-936.

Rabkin, J.M., Smith, M.J., Orloff, S.L., Corless, C.L., Stenzel, P. and Olyaei, A. (1999): Fatal fulminant hepatitis associated with bromfenac use. Ann. Pharmacother., 33, 945-947.

Raisanen, S.R., Lehenkari, P., Tasanen, M., Rahkila, P., Harkonen, P.L. and Vaananen, H.K. (1999): Carbonic anhydrase III protects cells from hydrogen peroxide-induced apoptosis. FASB J., 13, 513-522.

Recknagel, R.O., Glende, E.A., Dolak, J.A. and Waller, R.L. (1989): Mechanisms of carbon tetrachloride toxicity. Pharmac. Ther., 43, 139- 
154.

Ruepp, S.U., Tonge, R.P., Shaw, J., Wallis, N. and Pognan, F. (2002): Genomics and proteomics analysis of acetaminophen toxicity in mouse liver. Toxicol. Sci., 65, 135-150.

Salminen, W.F., Voellmy, R. and Roberts, S.M. (1997a): Differential heat shock protein induction by acetaminophen and a nonhepatotoxic regioisomer, 3'-hydroxyacetanilide, in mouse liver. J. Pharmacol. Exp. Ther., 282, 1533-1540.

Salminen, W.F., Voellmy, R. and Roberts, S.M. (1997b): Protection against hepatotoxicity by a single dose of amphetamine: The potential role of heat shock protein induction. Toxicol. Appl. Pharmacol., 147, 247-258.

Vella, A., de Groen, P.C. and Dinneen, S.F. (1998): Fetal hepatotoxicity associated with troglitazone. Ann. Intern. Med., 129, 1080.

Ward, J.H. (1963): Hierarchical grouping to optimize an objective function. J. Am. Stat. Assoc., 58,
236-244.

Waring, J.F., Jolly, R.A., Ciurlionis, R., Lum, P.Y., Praestgaard, J.T., Morfitt, D.C., Buratto, B., Roberts, C., Schadt, E. and Ulrich, R.G. (2001): Clustering of hepatotoxins based on mechanisms of toxicity using gene expression profiles. Toxicol. Appl. Pharmacol., 175, 28-42.

Watkins, P.B. (2005): Idiosyncratic liver injury: Challenges and approaches. Toxicol. Pathol., 33, 15.

Yamamoto, T., Kikkawa, R., Yamada, H. and Horii, I. (2005): Identification of oxidative stress-related proteins for predictive screening of hepatotoxicity using a proteomic approach. J. Toxicol. Sci., 30, 213-227.

Yoneda, T., Sato, M., Maeda, M. and Takagi, H. (1998): Identification of a novel adenylate kinase system in the brain: Cloning of the fourth adenylate kinase. Mol. Brain Res., 62, 187-195. 